Systematic Review

\title{
Polyunsaturated Fatty Acids and Chronic Pain: A Systematic Review and Meta-analysis
}

Jesús Prego-Domínguez, MSc, Fatine Hadrya, PhD, and Bahi Takkouche, MD, PhD

From: Universidade de Santiago de Compostela

Spain

Address Correspondence: Bahi Takkouche, MD, PhD Department of Preventive Medicine

University of Santiago de Compostela. 15782 Santiago de Compostela. Spain;

Centro de Investigación Biomédica en Red Epidemiología y Salud Pública (CIBER-ESP).

Av. Monforte de Lemos, 3-5. 28029 Madrid (Spain) E-mail: bahi.takkouche@usc.es

Disclaimer: There was no external funding in the preparation of this manuscript. Conflict of interest: Each author certifies that he or she, or a member of his or her immediate family, has no commercial association (i.e., consultancies, stock ownership, equity interest, patent/licensing arrangements, etc.) that might pose a conflict of interest in connection with the submitted manuscript.

Manuscript received: 11-13-2015 Revised manuscript received: 02-12-2016 Accepted for publication: 05-18-2016

Free full manuscript: www.painphysicianjournal.com
Background: Chronic pain is one of the most frequent disease symptoms and represents a global health problem with a considerable economic burden. The role of polyunsaturated fatty acids (PUFA) in chronic pain conditions was debated during the last decade with conflicting results.

Objective: To assess whether polyunsaturated fatty acids intake is useful as a preventive or curative tool in chronic pain.

Study Design: Systematic review and meta-analysis.

Setting: This study examined all published studies, either preventive or curative, on PUFA supplementation and chronic pain.

Methods: We retrieved studies published in any language by searching systematically Medline, Embase, Conference Proceedings Citation Index, dissertations databases, and the 5 regional bibliographic databases of the World Health Organization until May 2015.

We included both observational and intervention studies reporting effect measures and their confidence intervals of polyunsaturated fatty acids intake in the regular diet or supplementation and pain

Two investigators selected studies; extracted data independently on baseline characteristics, exposure, and outcomes; and rated the quality of interventional studies using Jadad score.

We calculated pooled standardized mean differences (SMDs) of pain indexes such as the Visual Analogue Score. We further carried out subgroup analyses by disease, type of PUFA, outcome scale, quality index, dose, and time of supplementation.

Results: We retrieved 5 observational and 46 intervention studies. Only one observational study showed a protective effect of PUFA. On the contrary, the interventional studies yielded a pooled random effects SMD of $-0.40(95 \% \mathrm{Cl}-0.58,-0.22)$, which indicates improvement, as 0 is the value that indicates absence of effect. The largest effect was found for dysmenorrhea (SMD $-0.82,95 \%$ $\mathrm{Cl}-1.21,-0.43), \Omega-3$ supplementation $(-0.47,95 \% \mathrm{Cl}-0.68,-0.26)$ and composite scores $(-0.58$ $95 \% \mathrm{Cl}-1.07,-0.09)$. Mitigation of pain was stronger for low doses $(-0.55,95 \% \mathrm{Cl}-0.79,-0.30)$ and short supplementation periods $(-0.56,95 \% \mathrm{Cl}-0.86,-0.25)$.

Limitations: While the number of curative studies was large, that of preventive studies available was limited.

Conclusion: Our results suggest that $\Omega-3$ PUFA supplementation moderately improves chronic pain, mainly that due to dysmenorrhea. Further investigation on the preventive potential of PUFA supplementation is needed, as the amount of evidence is scarce.

Key words: Meta-analysis, systematic review, chronic pain, PUFA, supplementation, $\Omega-3$, dysmenorrhea

Protocol registration: PROSPERO $n^{\circ}$ CRD42014010064

Pain Physician 2016; 19:521-535 
hronic pain represents an important global health problem. Its prevalence worldwide varies Jetween $10 \%$ and $25 \%$, and one-tenth of the adult population is diagnosed with chronic pain every year with a median duration of 7 years per episode (1). There is strong evidence that the contribution of chronic pain to the global burden of disease was, so far, underestimated. The World Health Organization (WHO) predicts that by 2030 the main contributors to the global burden of disease will be unipolar depression, coronary heart disease, cerebrovascular disease, and traffic accidents, all of which are associated with chronic pain as co-morbidity (2).

Furthermore, chronic pain implies a high cost which reaches $\$ 635$ billion annually in the USA and $€ 200$ billion in Europe (3), higher than that of cancer and cardiovascular diseases together (4).

The role of polyunsaturated fatty acids (PUFA) as an effective treatment for chronic pain conditions, such as rheumatoid arthritis and osteoarthritis, was debated in the last decade $(1,5,6) . \Omega$-3 PUFA eicosapentaenoic acid (EPA) and docosahexaenoic acid (DHA) decrease the activity of $\Omega-6$ PUFA arachidonic acid (ARA), a fatty acid that is related to the production of inflammatory mediators (7). So far, the results are conflicting, both in intervention and prevention studies, and range from improvement to absence of effect or even worsening of pain-producing conditions (8-11). Previous reviews, which used proxy measures of pain, have commented on the association between fatty acids intake and specific diseases in particular settings $(5,6,12)$. However, no comprehensive meta-analysis was carried out so far. We therefore summarized the scientific evidence through a systematic review and meta-analysis of the effect of PUFA's on pain syndromes.

\section{Methods}

We followed the PRISMA (Preferred Reporting Items for Systematic reviews and Meta-Analyses) guidelines (13). The review protocol was registered in PROSPERO (14).

\section{Search Strategy}

We searched MEDLINE, EMBASE, the 5 regional bibliographic databases of the World Health Organization (WHO) (African Index Medicus, Latin American and Caribbean Health Science Literature Database, Index Medicus for the Eastern Mediterranean Region, Index Medicus for South-East Asia Region, Western Pacific Region Index Medicus, the Conference Pro- ceedings Citation Index database, and the Open Access Thesis and Dissertations (OATD), from inception to May 2015. We used the algorithm, both in free text and Medical Subject Headings: PAIN AND (FATTY ACIDS OR OMEGA-3 OR PUFA) AND (CLINICAL TRIALS OR CASE-CONTROL OR COHORT) as the main search. Additionally, we performed searches with the key words in free text "abdominal pain," "low back pain," "joint pain," "headache"; and "fatty acids" to ensure that every study was retrieved. These key words were selected according to the most prevalent pain syndromes cited in the literature (15). We also reviewed the references of every article retrieved and those of recent reviews and monographs $(7,16)$.We did not include any language limitations. All searches were carried out independently by 2 researchers and the results were merged.

\section{Inclusion Criteria and Data Collection}

Studies were included if (1) they presented original data from observational or intervention studies, (2) the outcome was clearly defined as chronic pain, according to the definition of the International Association for the Study of Pain (17), (3) pain outcomes were measured through validated tools such as visual analogue scales (VAS) or composite scores for specific pain syndromes, (4) exposure was PUFA intake or supplementation, (5) they presented effect measures (means, mean difference, mean change) with their confidence intervals $(\mathrm{Cl})$, standard error, or enough data to calculate them (P-value, raw data, interquartile range).

We excluded studies on childhood pain and acute pain, and also those that did not specify the type of fatty acid. If data were duplicated in more than one study, the most recent one was used. When an article reported results for more than one pain-PUFA relationship (i.e., different supplements), we considered each one as a separate study unit. When necessary, we contacted authors for further information $(9,10,18)$. The studies retrieved were divided into 2 groups, according to their preventive or curative purpose.

We developed a structured questionnaire to extract the following data: (1) author, (2) year of publication, (3) country, (4) study design, (5) sample size, (6) disease, (7) exposure (intervention group and comparison group), (8) pain measurement instrument, (9) effect measure, and (10) adjustment factors for observational studies. Additionally, for clinical trials, we extracted: (11) intervention period and (12) study duration. 


\section{Quality Assessment}

The quality of clinical trials was assessed using the Jadad index which rates aspects of randomization, blinding, and withdrawals (19). A score of 3 or higher out of 5 was considered as "low risk of bias."

Both the extraction process and the quality rating were performed by 2 researchers independently, and results were merged by consensus. Due to the small number of the studies retrieved ( 5 with 3 different designs) and thus, the impossibility of performing a metaanalysis, we did not assess the quality of observational studies through a standard scale.

\section{Data Synthesis and Analysis}

Study-specific standardized mean differences (SMDs) were weighted by the inverse of their variance to compute a pooled effect size and its $95 \% \mathrm{Cl}$.

SMDs are used as summary statistics when all the studies assess the same outcome but measure it in a variety of ways (as in the case of different measurement scores). SMDs compare treatment and placebo and are weighted by the pooled standard deviation (SD) of both arms. An SMD of zero means no difference between treatment and placebo, a negative result favors treatment in our case (as the pain score decreases when treatment is effective) and a positive result favors placebo. A large effect is defined as $>0.8$ unit, a medium effect as $>0.5$ unit, and a small effect as $<0.2$ units (20). When all the information required was not available, we assumed normal distribution of SMDs and used median and interquartile range to calculate means and SDs. Furthermore, when a study reported different doses of the same supplement, or included pain from different anatomical sites (e.g., joint and abdominal pain), we first pooled its various SMDs and included this data in the main analysis. We later stratified our results by subgroup.

We assessed heterogeneity using Cochran's Qstatistics and the proportion of the total variance due to between-study variance 12 . We present both fixed effects and random effects pooled estimates, but used the latter when heterogeneity was present.

\section{Cumulative Meta-analysis}

In addition to the general meta-analysis, we performed a cumulative meta-analysis by date of publication both for all pain outcomes and for rheumatoid arthritis. The objective was to detect a possible trend in the estimation.

\section{Subgroup Analyses}

In addition to the main analysis of studies of fatty acid supplementation and chronic pain, we carried out subgroup analyses by disease (rheumatoid arthritis, dysmenorrhoea, mastalgia, other chronic pain syndromes), type of fatty acid supplementation $(\Omega-3, \Omega-6$, dietary intervention/supplement, combined), outcome scale (VAS/Likert scale, composite score), and Jadad score (low, high).

Further subgroup analysis includes studies administering $\Omega-3$ supplementation up to 3 months and longer than 3 months. These intervals were based on previous reports suggesting that the therapeutic effects of $\Omega-3$ PUFAs are observed after approximately 3 months (5). We also stratified by daily dose of EPA $(<1.35$ and $>$ $1.35 \mathrm{~g} /$ day). We chose this threshold because the effect on pain was observed in the interval $1.35-2.7 \mathrm{~g} /$ day in previous studies (21). When doses were given in $\mathrm{mg} /$ $\mathrm{kg} /$ day, we calculated the total daily dose for an adult of $70 \mathrm{~kg}$.

All subgroups analyses were planned a priori.

\section{Publication Bias}

We used funnel plots to detect publication bias and, more formally, Egger's regression test (22), and the Trim-and-Fill method to correct for potential publication bias (23).

Statistical analysis was performed with Comprehensive Meta-analysis software (Biostat, Englewood, NJ).

\section{Results}

Our search brought 47 articles which provided data on 51 studies: 5 observational studies $(10,11,24-26)$ and 46 intervention studies $(8,9,18,27-65)$. The complete search strategy and exclusion motives are shown in Fig. 1. The main characteristics of the studies included are shown in Table 1. We performed separate analyses for observational studies and intervention studies. As the number of preventive studies was scarce and different designs were used, we were unable to carry out a meta-analysis. Therefore, in this part, we limited our study to a narrative review.

\section{Observational Studies}

We found 5 observational studies ( 3 cross-sectional, one case-control, and one cohort study), carried out in 4 countries, that met our inclusion criteria. The data from one study were obtained from the authors (10).

In Denmark in 1995, Deutch (11) carried out a 
Pain Physician: November/December 2016: 19:521-535

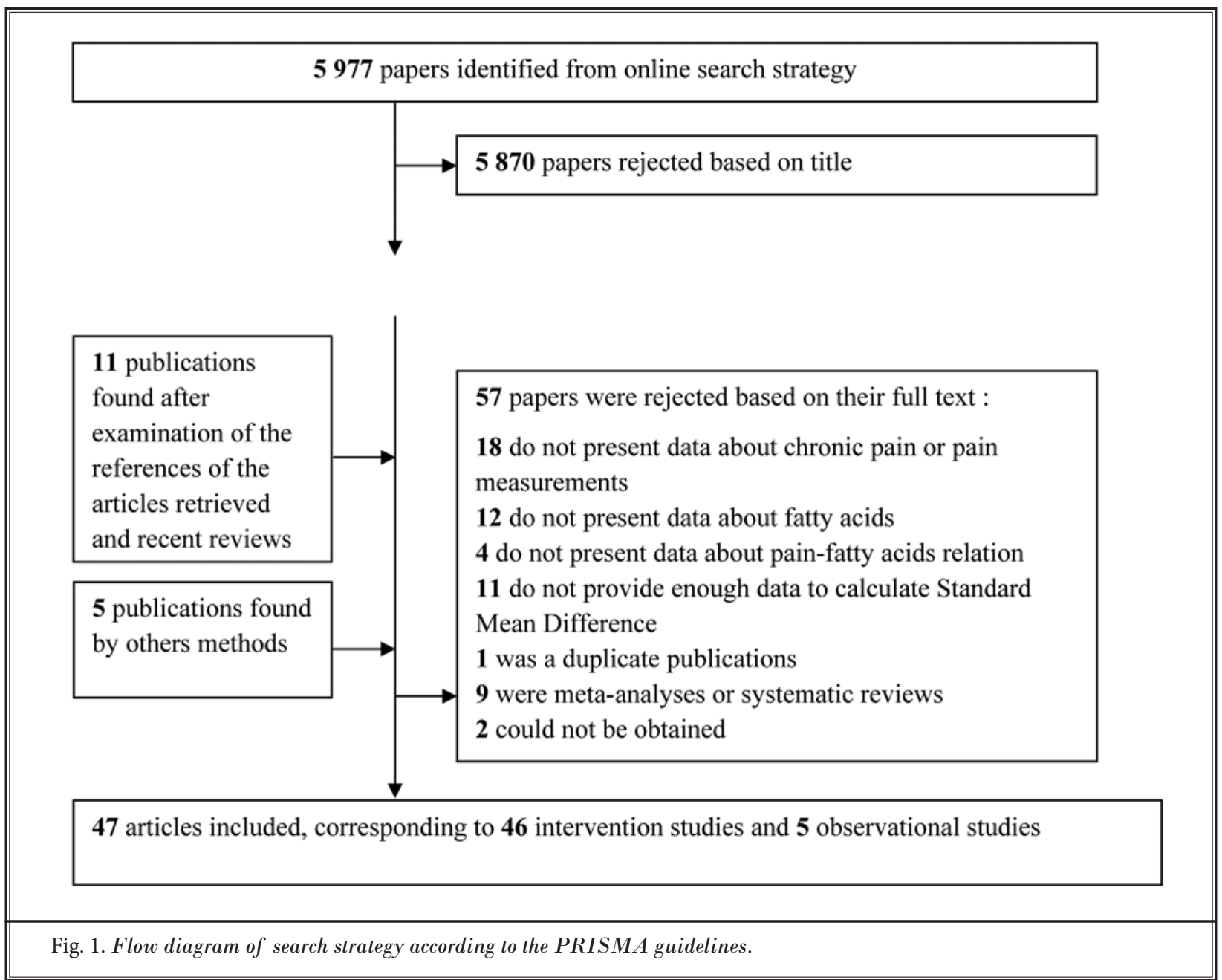

cross-sectional study among 181 healthy volunteers about menstrual pain and dietary habits using a self-administered questionnaire distributed at the beginning of the study and 6 months later. The author reported a strong association between increased pain and a low intake of $\Omega-3$ fatty acids from fish or fish oils ( $r=0.53$ ) and between increased pain and a low ratio of $\Omega-3$ to $\Omega-6$ fatty acids in the diet $(r=0.42)$.

Two other cross-sectional studies were carried out by Nagata et al in Japan in 2004 and $2005(24,25)$ among nursing students on dietary habits and premenstrual syndrome and menstrual pain, with 189 and 276 participants, respectively. In both studies no association was found between PUFA intake and any of the syndromes.

In 1997, Di Cintio et al (26) carried out a case-control study in Italy on dietary habits, reproductive and menstrual factors, and risk of dysmenorrhoea. Dietary habits were assessed by trained interviewers using a standard questionnaire in 106 cases and 145 controls recruited at an outpatient gynecological clinic. These authors did not find any association between $\Omega-3$ or $\Omega-6$ intake and dysmenorrhoea.

Finally, in 2011 a Spanish cohort study on dietary habits and quality of life, with 4 years of follow-up and 8,430 participants showed no association between PUFA intake and bodily pain or other SF-36 questionnaire domains related to pain (10). To summarize, only one cross-sectional study out of these 5 observational studies found a protective effect of fatty acids on pain.

\section{Intervention Studies}

We found 46 studies (45 clinical trials and one quasi-experimental study) carried out in 18 different countries. The majority of the studies was on rheuma- 
Table 1. Main characteristics of the studies.

\begin{tabular}{|c|c|c|c|c|c|c|c|c|c|}
\hline \multicolumn{10}{|c|}{ Observational Studies } \\
\hline $\begin{array}{l}\text { Study } \\
\text { (year) }\end{array}$ & Country & Design & Disease & Population & $\begin{array}{l}\text { Sample } \\
\text { size }\end{array}$ & Exposure & $\begin{array}{l}\text { Measure } \\
\text { time }\end{array}$ & Outcome score & $\begin{array}{c}\text { Impairment/ } \\
\text { Adjust/Restriction } \\
\text { factors }\end{array}$ \\
\hline $\begin{array}{l}\text { Ruano et } \\
\text { al (2011) }\end{array}$ & Spain & Cohort & Bodily pain & University & $\begin{array}{l}15,089 \\
\text { entered, } \\
8,430 \\
\text { included }\end{array}$ & $\begin{array}{l}\text { Dietary fatty } \\
\text { acid intake } \\
\text { (saturated } \\
\text { and trans- } \\
\text { unsaturated } \\
\text { fats, MUFAs } \\
\text { and PUFAs) }\end{array}$ & $\begin{array}{l}\text { Twice }(0- \\
4 \text { years })\end{array}$ & $\begin{array}{l}\text { SF-36 Health } \\
\text { Survey }\end{array}$ & $\begin{array}{l}\text { Age, sex, smoking, } \\
\text { leisure time } \\
\text { physical activity, } \\
\text { total energy } \\
\text { intake, BMI, } \\
\text { Mediterranean Diet } \\
\text { Score }\end{array}$ \\
\hline $\begin{array}{l}\text { Nagata } \\
\text { et al } \\
(2005 a)\end{array}$ & Japan & $\begin{array}{l}\text { Cross- } \\
\text { sectional }\end{array}$ & $\begin{array}{l}\text { Premenstrual } \\
\text { syndrome }\end{array}$ & $\begin{array}{l}\text { University - } \\
\text { nursing school }\end{array}$ & $\begin{array}{l}315 \\
\text { entered, } \\
189 \\
\text { included }\end{array}$ & $\begin{array}{l}\text { Dietary fatty } \\
\text { acid intake } \\
\text { (saturated fats, } \\
\text { MUFAs and } \\
\text { PUFAs) }\end{array}$ & Once & MDQ score & $\begin{array}{l}\text { Age, total energy } \\
\text { intake, marital } \\
\text { status, smoking } \\
\text { status, exercise, } \\
\text { age at menarche, } \\
\text { number of days of } \\
\text { bleeding }\end{array}$ \\
\hline $\begin{array}{l}\text { Nagata } \\
\text { et al } \\
(2005 b)\end{array}$ & Japan & $\begin{array}{l}\text { Cross- } \\
\text { sectional }\end{array}$ & Menstrual pain & $\begin{array}{l}\text { University - } \\
\text { nursing school }\end{array}$ & $\begin{array}{l}362 \\
\text { entered, } \\
276 \\
\text { included }\end{array}$ & $\begin{array}{l}\text { Dietary fatty } \\
\text { acid intake } \\
\text { (saturated fats, } \\
\text { MUFAs and } \\
\text { PUFAs) }\end{array}$ & Once & $\begin{array}{l}\text { Verbal } \\
\text { multidimensional } \\
\text { scoring system }\end{array}$ & $\begin{array}{l}\text { Age, total energy } \\
\text { intake, smoking } \\
\text { status, age at } \\
\text { menarche }\end{array}$ \\
\hline $\begin{array}{l}\text { Di Cintio } \\
\text { et al } \\
(1997)\end{array}$ & Italy & $\begin{array}{l}\text { Case- } \\
\text { control } \\
\text { study }\end{array}$ & Dysmenorrhea & $\begin{array}{l}\text { Outpatient } \\
\text { clinic }\end{array}$ & $\begin{array}{l}106 \text { cases } \\
\text { and } 145 \\
\text { controls }\end{array}$ & $\begin{array}{l}\text { Nutritional } \\
\text { habits (whole } \\
\text { foods, fats and } \\
\text { condiments } \\
\text { consumption) }\end{array}$ & Once & $\begin{array}{l}\text { Andersch } \\
\text { and Milsom's } \\
\text { classification, VAS }\end{array}$ & $\begin{array}{l}\text { Age, education, } \\
\text { smoking, selected } \\
\text { menstrual } \\
\text { characteristics, } \\
\text { reproductive } \\
\text { history }\end{array}$ \\
\hline $\begin{array}{l}\text { Deutch } \\
\text { (1995) }\end{array}$ & Denmark & $\begin{array}{l}\text { Cross- } \\
\text { sectional } \\
\text { cohort }\end{array}$ & Menstrual pain & $\begin{array}{l}\text { Community } \\
\text { based } \\
\text { - volunteers }\end{array}$ & $\begin{array}{l}44 \text { cases } \\
\text { and } 137 \\
\text { controls }\end{array}$ & $\begin{array}{l}\text { Dietary fatty } \\
\text { acid intake } \\
\text { (saturated fats } \\
\text { and PUFAs) }\end{array}$ & $\begin{array}{l}\text { Twice (0 - } \\
6 \text { months) }\end{array}$ & $\begin{array}{l}\text { Self-administered } \\
\text { questionnaire }\end{array}$ & $\begin{array}{l}\text { Parity, time of food } \\
\text { record, total energy } \\
\text { intake. }\end{array}$ \\
\hline
\end{tabular}

\begin{tabular}{|c|c|c|c|c|c|c|c|c|c|}
\hline \multicolumn{10}{|c|}{ Intervention Studies } \\
\hline Study (year) & Country & $\begin{array}{l}\text { Intervention } \\
\text { period }\end{array}$ & Disease & $\begin{array}{c}\text { Sample size } \\
\text { (Treatment/ } \\
\text { Control) }\end{array}$ & Intervention & Daily dose & Comparison & $\begin{array}{l}\text { Outcome } \\
\text { score }\end{array}$ & $\begin{array}{l}\text { Standard } \\
\text { Mean } \\
\text { Difference } \\
(95 \% \mathrm{CI})\end{array}$ \\
\hline $\begin{array}{l}\text { Lopes Cortes } \\
\text { (2013) }\end{array}$ & Brazil & 12 weeks & $\begin{array}{l}\text { Myofascial pain } \\
\text { syndrome }\end{array}$ & $41(20 / 21)$ & $\Omega-3$ & $\begin{array}{l}1080 \mathrm{mg} \\
\mathrm{EPA}, 720 \\
\mathrm{mg} \text { DHA }\end{array}$ & - & VAS & $\begin{array}{l}-0.87(-1.38, \\
-0.35)\end{array}$ \\
\hline $\begin{array}{l}\text { Park et al } \\
\text { (2013) }\end{array}$ & Korea & 16 weeks & $\begin{array}{l}\text { Rheumatoid } \\
\text { arthritis }\end{array}$ & $109(41 / 40)$ & $\Omega-3$ & $\begin{array}{l}2090 \mathrm{mg} \\
\text { EPA, } 1160 \\
\text { mg DHA }\end{array}$ & Sunflower oil & VAS & $\begin{array}{l}0.30(-0.14 \\
0.74)\end{array}$ \\
\hline $\begin{array}{l}\text { Sohrabi et al } \\
\text { (2013) }\end{array}$ & Iran & 3 months & $\begin{array}{l}\text { Premenstrual } \\
\text { syndrome }\end{array}$ & $139(63 / 61)$ & $\begin{array}{l}\Omega-3(1 \mathrm{~m}), 8 \\
\text { days before } \\
\text { and } 2 \text { after } \\
\text { menstruation } \\
(2 \mathrm{~m})\end{array}$ & $\begin{array}{l}360 \mathrm{mg} \\
\text { EPA, 240 } \\
\text { mg DHA }\end{array}$ & Placebo & VAS & $\begin{array}{l}-0.41(-0.76 \text {, } \\
-0.05)\end{array}$ \\
\hline $\begin{array}{l}\text { Ramsden et al } \\
\text { (2013) }\end{array}$ & USA & 12 weeks & $\begin{array}{l}\text { Chronic } \\
\text { headaches }\end{array}$ & $67(33 / 34)$ & $\begin{array}{l}\text { Dietary } \\
\text { High } \Omega-3 \\
+ \text { low } \Omega-6 \\
\text { intervention }\end{array}$ & - & Low $\Omega-6$ & HIT-6 & $\begin{array}{l}-0.94(-1.45, \\
-0.44)\end{array}$ \\
\hline
\end{tabular}


Pain Physician: November/December 2016: 19:521-535

Table 1. Main characteristics of the studies (continued).

\begin{tabular}{|c|c|c|c|c|c|c|c|c|c|}
\hline \multicolumn{10}{|c|}{ Intervention Studies } \\
\hline Study (year) & Country & $\begin{array}{l}\text { Intervention } \\
\text { period }\end{array}$ & Disease & $\begin{array}{c}\text { Sample size } \\
\text { (Treatment/ } \\
\text { Control) }\end{array}$ & Intervention & Daily dose & Comparison & $\begin{array}{l}\text { Outcome } \\
\text { score }\end{array}$ & $\begin{array}{l}\text { Standard } \\
\text { Mean } \\
\text { Difference } \\
(95 \% \mathrm{CI})\end{array}$ \\
\hline $\begin{array}{l}\text { Rahbar et al } \\
(2012)\end{array}$ & Iran & 3 months & $\begin{array}{l}\text { Primary } \\
\text { dysmenorrhea }\end{array}$ & $100(95 / 95)$ & $\Omega-3$ & $\begin{array}{l}180 \mathrm{mg} \\
\text { EPA, 120 } \\
\text { mg DHA }\end{array}$ & Placebo & $\begin{array}{l}\text { Cox } \\
\text { menstrual } \\
\text { symptom } \\
\text { scale }\end{array}$ & $\begin{array}{l}-1.22(-1.53, \\
-0.91)\end{array}$ \\
\hline $\begin{array}{l}\text { Caturla et al } \\
\text { (2011) }\end{array}$ & Spain & 9 weeks & $\begin{array}{l}\text { Osteoarthritis } \\
\text { / Reumatoid } \\
\text { arthritis }\end{array}$ & $45(19 / 12)$ & $\Omega-3$ & $\begin{array}{l}222 \mathrm{mg} \\
\mathrm{EPA}, 178 \\
\mathrm{mg} \text { DHA } \\
\text { ( } 5 \mathrm{wk} \text { ) and } \\
111 \mathrm{mg} \\
\mathrm{EPA}, 89 \\
\text { DHA (4 } \\
\text { wk }\end{array}$ & Placebo & $\begin{array}{l}\text { WOMAC } \\
\text { score }\end{array}$ & $\begin{array}{l}-1.43(-2.24, \\
-0.63)\end{array}$ \\
\hline $\begin{array}{l}\text { Guimarães et } \\
\text { al (2011) }\end{array}$ & Brazil & 28 days & $\begin{array}{l}\text { Centrally } \\
\text { mediated } \\
\text { chronic myalgia }\end{array}$ & $24(12 / 12)$ & $\begin{array}{l}\text { Splint + fish } \\
\text { oil }\end{array}$ & $\begin{array}{l}3 \mathrm{~g} \text { (no } \\
\mathrm{EPA} / \mathrm{DHA} \\
\text { doses } \\
\text { given) }\end{array}$ & $\begin{array}{l}\text { Splint }+ \\
\text { avoid foods } \\
\text { rich in } \Omega-3\end{array}$ & VAS & $\begin{array}{l}0.32(-0.49, \\
1.12)\end{array}$ \\
\hline $\begin{array}{l}\text { Kawabata et al } \\
\text { (2011) }\end{array}$ & Japan & 4 weeks & $\begin{array}{l}\text { Asthenopia } \\
\text { (eye-pain, low } \\
\text { back pain, } \\
\text { headache) }\end{array}$ & $22(11 / 9)$ & Fish oil & $\begin{array}{l}162 \mathrm{mg} \\
\text { EPA, 783 } \\
\text { mg DHA }\end{array}$ & $\begin{array}{l}\text { Middle chain } \\
\text { triglycerides } \\
\text { (edible oil) }\end{array}$ & VAS & $\begin{array}{l}-0.75(-1.66, \\
0.16)\end{array}$ \\
\hline $\begin{array}{l}\text { Moghadamnia } \\
\text { et al (2010) }\end{array}$ & Iran & 3 months & Dysmenorrhea & $36(36 / 36)$ & Fish oil & $\begin{array}{l}550 \mathrm{mg} \\
\text { EPA, 205 } \\
\text { mg DHA }\end{array}$ & $\begin{array}{l}\text { Vitamins } \\
\text { emulsion }\end{array}$ & VAS & $\begin{array}{l}-0.81(-1.29, \\
-0.33)\end{array}$ \\
\hline $\begin{array}{l}\text { Pruthi et al } \\
\text { (2010) }\end{array}$ & USA & 6 months & Mastalgia & $43(21 / 22)$ & $\begin{array}{l}\mathrm{EPO}+\text { corn } \\
\text { oil }\end{array}$ & $\begin{array}{l}315 \mathrm{mg} \\
\text { GLA }\end{array}$ & Corn oil & VAS & $\begin{array}{l}0.18(-0.41, \\
0.78)\end{array}$ \\
\hline $\begin{array}{l}\text { Das Gupta et } \\
\text { al (2009) }\end{array}$ & Bangladesh & 12 weeks & $\begin{array}{l}\text { Rheumatoid } \\
\text { arthritis }\end{array}$ & $100(40 / 41)$ & $\begin{array}{l}\text { Indomethacin } \\
+\Omega-3\end{array}$ & $\begin{array}{l}540 \mathrm{mg} \\
\mathrm{EPA}, 360 \\
\mathrm{mg} \text { DHA }\end{array}$ & Indomethacin & VAS & $\begin{array}{l}-1.07(-1.54, \\
-0.60)\end{array}$ \\
\hline $\begin{array}{l}\text { Gruenwald et } \\
\text { al (2009) }\end{array}$ & Germany & 26 weeks & Osteoarthritis & $177(90 / 87)$ & $\begin{array}{l}\text { Glucosamine } \\
\text { sulfate + Fish } \\
\text { oil }+\Omega-3\end{array}$ & $\begin{array}{l}1332 \mathrm{mg} \\
\text { Fish oil, } \\
600 \mathrm{mg} \Omega-3 \\
\text { (no EPA/ } \\
\text { DHA dose } \\
\text { given) }\end{array}$ & $\begin{array}{l}\text { Glucosamine } \\
\text { sulfate + } \\
\text { mixture oils }\end{array}$ & $\begin{array}{l}\text { WOMAC } \\
\text { score }\end{array}$ & $\begin{array}{l}-0.26(-0.56 \text {, } \\
0.03)\end{array}$ \\
\hline $\begin{array}{l}\text { Aryaeian et al } \\
(2009)\end{array}$ & Iran & 12 weeks & $\begin{array}{l}\text { Rheumatoid } \\
\text { arthritis }\end{array}$ & $52(22 / 22)$ & $\begin{array}{l}\text { Conjugated } \\
\text { linoleic acid }\end{array}$ & $2500 \mathrm{mg}$ & $\begin{array}{l}\text { High oleic } \\
\text { sunflower oil }\end{array}$ & VAS & $\begin{array}{l}1.41(0.7 \\
2.07)\end{array}$ \\
\hline $\begin{array}{l}\text { Galarraga et al } \\
\text { (2008) }\end{array}$ & $\begin{array}{l}\text { United } \\
\text { Kingdom }\end{array}$ & 9 months & $\begin{array}{l}\text { Rheumatoid } \\
\text { arthritis }\end{array}$ & $97(49 / 48)$ & $\begin{array}{l}\text { Cod liver oil + } \\
\text { fish oil }\end{array}$ & $\begin{array}{l}1500 \mathrm{mg} \\
\mathrm{EPA}, 700 \\
\mathrm{mg} \text { DHA }\end{array}$ & $\begin{array}{l}\text { Identical air- } \\
\text { filled placebo }\end{array}$ & VAS & $\begin{array}{l}-0.32(-0.72 \text {, } \\
0.08)\end{array}$ \\
\hline $\begin{array}{l}\text { Goyal et al } \\
(2005)\end{array}$ & $\begin{array}{l}\text { United } \\
\text { Kingdom }\end{array}$ & 12 months & Mastalgia & $\begin{array}{l}278 \\
(140 / 138)\end{array}$ & $\mathrm{EPO}$ & $\begin{array}{l}320 \mathrm{mg} \\
\text { GLA }\end{array}$ & Coconut oil & $\begin{array}{l}\text { Breast } \\
\text { Pain } \\
\text { Score }\end{array}$ & $\begin{array}{l}-0.03(-0.27, \\
0.20)\end{array}$ \\
\hline \multirow[t]{2}{*}{$\begin{array}{l}\text { Berbert et al } \\
(2005)\end{array}$} & \multirow[t]{2}{*}{ Brazil } & \multirow[t]{2}{*}{24 weeks } & \multirow[t]{2}{*}{$\begin{array}{l}\text { Rheumatoid } \\
\text { arthritis }\end{array}$} & $55(17 / 13)$ & $\begin{array}{l}\text { Fish oil } \Omega-3+ \\
\text { olive oil }\end{array}$ & $\begin{array}{l}1800 \mathrm{mg} \\
\mathrm{EPA}, 1200 \\
\mathrm{mg} \mathrm{DHA} \text {, } \\
6.8 \mathrm{~g} \mathrm{OA}\end{array}$ & \multirow[t]{2}{*}{ Soy oil } & \multirow[t]{2}{*}{$\begin{array}{l}\text { 5-point } \\
\text { score }\end{array}$} & $\begin{array}{l}-1.32(-2.12 \text {, } \\
-0.53)\end{array}$ \\
\hline & & & & $55(13 / 13)$ & Fish oil $\Omega-3$ & $\begin{array}{l}1800 \mathrm{mg} \\
\mathrm{EPA}, 1200 \\
\mathrm{mg} \text { DHA }\end{array}$ & & & $\begin{array}{l}-0.60(-1.38, \\
0.19)\end{array}$ \\
\hline $\begin{array}{l}\text { Sundrarjun et } \\
\text { al (2004) }\end{array}$ & Thailand & 12 weeks & $\begin{array}{l}\text { Rheumatoid } \\
\text { arthritis }\end{array}$ & $46(23 / 23)$ & $\begin{array}{l}\text { Low } \Omega-6 \text { diet } \\
+ \text { fish oil }\end{array}$ & $\begin{array}{l}1880 \mathrm{mg} \\
\mathrm{EPA}, 1480 \\
\mathrm{mg} \text { DHA }\end{array}$ & $\begin{array}{l}\text { Low } \Omega-6 \text { diet } \\
+ \text { Placebo }\end{array}$ & VAS & $\begin{array}{l}0.24(-0.34, \\
0.82)\end{array}$ \\
\hline
\end{tabular}


PUFA and Pain: A Meta-analysis

Table 1. Main characteristics of the studies (continued).

\begin{tabular}{|c|c|c|c|c|c|c|c|c|c|}
\hline \multicolumn{10}{|c|}{ Intervention Studies } \\
\hline Study (year) & Country & $\begin{array}{l}\text { Intervention } \\
\text { period }\end{array}$ & Disease & $\begin{array}{c}\text { Sample size } \\
\text { (Treatment/ } \\
\text { Control) }\end{array}$ & Intervention & Daily dose & Comparison & $\begin{array}{l}\text { Outcome } \\
\text { score }\end{array}$ & $\begin{array}{c}\text { Standard } \\
\text { Mean } \\
\text { Difference } \\
(95 \% \mathrm{CI})\end{array}$ \\
\hline $\begin{array}{l}\text { Remans et al } \\
\text { (2004) }\end{array}$ & Netherlands & 4 months & $\begin{array}{l}\text { Rheumatoid } \\
\text { arthritis }\end{array}$ & $66(26 / 29)$ & $\begin{array}{l}\text { PUFA } \\
\text { supplement } \\
\text { drink }\end{array}$ & $\begin{array}{l}1400 \mathrm{mg} \\
\text { EPA, 200 } \\
\text { mg DHA } \\
-500 \mathrm{mg} \\
\text { GLA }\end{array}$ & Placebo drink & VAS & $\begin{array}{l}0.51(-0.02, \\
1.05)\end{array}$ \\
\hline $\begin{array}{l}\text { Sampalis et al } \\
\text { (2003) }\end{array}$ & Canada & 3 months & $\begin{array}{l}\text { Premenstrual } \\
\text { syndrome } \\
\text { and } \\
\text { dysmenorrhea }\end{array}$ & $70(36 / 34)$ & $\begin{array}{l}\text { KO daily } \\
(1 \mathrm{~m}) \text { and } 8 \\
\text { days before } \\
\text { and } 2 \text { after } \\
\text { menstruation } \\
(2 \mathrm{~m})\end{array}$ & $\begin{array}{l}284 \mathrm{mg} \\
\text { EPA, 170 } \\
\text { mg DHA }\end{array}$ & $\Omega-3$ fish oil & VAS & $\begin{array}{l}-0.82(-1.31, \\
-0.33)\end{array}$ \\
\hline $\begin{array}{l}\text { Guerra dos } \\
\text { Santos (2003) }\end{array}$ & Brazil & 3 months & Mastalgia & $111(56 / 55)$ & Borage oil & $\begin{array}{l}326 \mathrm{mg} \mathrm{LA}, \\
180 \mathrm{mg} \\
\text { GLA }\end{array}$ & Sunflower oil & $\begin{array}{l}\text { Cardiff } \\
\text { Breast } \\
\text { Score }\end{array}$ & $\begin{array}{l}0.11(-0.26 \text {, } \\
0.49)\end{array}$ \\
\hline \multirow[t]{2}{*}{$\begin{array}{l}\text { Blommers et al } \\
(2002)\end{array}$} & \multirow[t]{2}{*}{ Netherlands } & \multirow[t]{2}{*}{6 months } & \multirow[t]{2}{*}{ Mastalgia } & $90(30 / 30)$ & $\begin{array}{l}\mathrm{EPO}+\text { corn } \\
\text { oil + wheat- } \\
\text { germ oil }\end{array}$ & $\begin{array}{l}288 \mathrm{mg} \\
\text { GLA, } 2136 \\
\text { mg LA }\end{array}$ & \multirow[t]{2}{*}{$\begin{array}{l}\text { Corn oil + } \\
\text { wheat-germ } \\
\text { oil }\end{array}$} & \multirow[t]{2}{*}{$\begin{array}{l}\text { 4-point } \\
\text { score }\end{array}$} & $\begin{array}{l}0.05(-0.45, \\
0.56)\end{array}$ \\
\hline & & & & $90(30 / 30)$ & $\begin{array}{l}\text { Fish oil + } \\
\text { corn oil }\end{array}$ & $\begin{array}{l}1128 \mathrm{mg} \\
\text { EPA, 714 } \\
\text { mg DHA }\end{array}$ & & & $\begin{array}{l}0.00(-0.51, \\
0.51)\end{array}$ \\
\hline \multirow[t]{2}{*}{$\begin{array}{l}\text { Adam et al } \\
(2002)\end{array}$} & \multirow[t]{2}{*}{ Germany } & \multirow[t]{2}{*}{3 months } & \multirow[t]{2}{*}{$\begin{array}{l}\text { Rheumatoid } \\
\text { arthritis }\end{array}$} & $60(30 / 30)$ & $\begin{array}{l}\text { Fish oil } \\
+ \text { anti- } \\
\text { inflammatory } \\
\text { diet }\end{array}$ & $\begin{array}{l}128.1 \mathrm{mg} \\
\mathrm{EPA}, 95.8 \\
\mathrm{mg} \text { DHA } \\
/ 10 \mathrm{~kg} \text { of } \\
\text { body weight }\end{array}$ & \multirow[t]{2}{*}{ Corn oil } & \multirow[t]{2}{*}{ VAS } & $\begin{array}{l}-1.92(-2.54, \\
-1.31)\end{array}$ \\
\hline & & & & $60(30 / 30)$ & $\begin{array}{l}\text { Fish oil + } \\
\text { standard } \\
\text { western diet }\end{array}$ & $\begin{array}{l}128.1 \mathrm{mg} \\
\mathrm{EPA}, 95.8 \\
\mathrm{mg} \text { DHA } \\
/ 10 \mathrm{~kg} \text { of } \\
\text { body weight }\end{array}$ & & & $\begin{array}{l}-0.96(-1.50 \\
-0.43)\end{array}$ \\
\hline $\begin{array}{l}\text { Harel et al } \\
(2002)\end{array}$ & USA & 2 months & Migraines & $27(23 / 23)$ & $\begin{array}{l}\text { Marine } \Omega-3 \\
\text { ethyl ester } \\
\text { concentrate }\end{array}$ & $\begin{array}{l}756 \mathrm{mg} \\
\text { EPA, 498 } \\
\text { mg DHA }\end{array}$ & $\begin{array}{l}\text { Olive oil } \\
\text { ethyl ester } \\
\text { concentrate }\end{array}$ & $\begin{array}{l}\text { 7-point } \\
\text { score }\end{array}$ & $\begin{array}{l}-1.32(-1.96 \\
-0.69)\end{array}$ \\
\hline $\begin{array}{l}\text { Volker et al } \\
(2000)\end{array}$ & Australia & 15 weeks & $\begin{array}{l}\text { Rheumatoid } \\
\text { arthritis }\end{array}$ & $50(13 / 13)$ & $\begin{array}{l}\text { Fish oil }(60 \% \\
\Omega-3)\end{array}$ & $\begin{array}{l}40 \mathrm{mg} / \\
\mathrm{kg} / \mathrm{d} \text { of fish } \\
\text { oil (50\% } \\
\text { EPA, } 35 \% \\
\text { DHA) }\end{array}$ & $\begin{array}{l}50 / 50 \text { corn/ } \\
\text { olive oil }\end{array}$ & VAS & $\begin{array}{l}-0.01(-0.78, \\
0.76)\end{array}$ \\
\hline $\begin{array}{l}\text { Sarzi-Puttini et } \\
\text { al (2000) }\end{array}$ & Italy & 24 weeks & $\begin{array}{l}\text { Rheumatoid } \\
\text { arthritis }\end{array}$ & $50(22 / 21)$ & $\begin{array}{l}\text { Diet rich in } \\
\text { unsaturated } \\
\text { fat }+ \\
\text { hipoallergenic } \\
\text { foods }\end{array}$ & $\begin{array}{l}2: 1 \text { ratio of } \\
\text { unsaturated } \\
\text { to saturated } \\
\text { fat }\end{array}$ & Normal diet & VAS & $\begin{array}{l}-0.16(-0.76 \\
0.44)\end{array}$ \\
\hline $\begin{array}{l}\text { Hansen et al } \\
\text { (1996) }\end{array}$ & Denmark & 6 months & $\begin{array}{l}\text { Rheumatoid } \\
\text { arthritis }\end{array}$ & $109(36 / 45)$ & $\begin{array}{l}\text { Adjusted } \\
\text { Graastener } \\
\text { diet ( } 800 \mathrm{~g} \\
\text { fresh fish } \\
\text { a week } \\
\text { OR fish oil } \\
\text { supplement) }\end{array}$ & $\begin{array}{l}600 \mathrm{mg} \\
\text { EPA, } 420 \\
\text { mg DHA }\end{array}$ & Normal diet & VAS & $\begin{array}{l}-0.37(-0.81, \\
0.08)\end{array}$ \\
\hline $\begin{array}{l}\text { Zurier et al } \\
(1996)\end{array}$ & USA & 12 months & $\begin{array}{l}\text { Rheumatoid } \\
\text { arthritis }\end{array}$ & $56(22 / 19)$ & $\begin{array}{l}\text { Borage seed } \\
\text { oil }\end{array}$ & $\begin{array}{l}2800 \mathrm{mg} \\
\text { GLA }\end{array}$ & $\begin{array}{l}\text { Sunflower } \\
\text { seed oil }\end{array}$ & VAS & $\begin{array}{l}-0.96(-1.61, \\
-0.31)\end{array}$ \\
\hline $\begin{array}{l}\text { Kremer et al } \\
\text { (1995) }\end{array}$ & USA & 48 weeks & $\begin{array}{l}\text { Rheumatoid } \\
\text { arthritis }\end{array}$ & $66(15 / 14)$ & $\begin{array}{l}\text { Diclofenac } \\
+ \text { fish oil + } \\
\text { corn oil }\end{array}$ & $\begin{array}{l}130 \mathrm{mg} / \\
\mathrm{kg} / \mathrm{d} \text { of } \\
\Omega-3(44 \% \\
\text { EPA }-24 \% \\
\text { DHA })\end{array}$ & $\begin{array}{l}\text { Diclofenac + } \\
\text { Corn oil }\end{array}$ & $\begin{array}{l}\text { 5-point } \\
\text { score }\end{array}$ & $\begin{array}{l}0.14(-0.59, \\
0.87)\end{array}$ \\
\hline $\begin{array}{l}\text { Nordstrom et } \\
\text { al (1995) }\end{array}$ & Finland & 3 months & $\begin{array}{l}\text { Rheumatoid } \\
\text { arthritis }\end{array}$ & $22(11 / 11)$ & Flaxseed oil & $\begin{array}{l}9600 \mathrm{mg} \\
a-\mathrm{LNA}\end{array}$ & Safflower oil & VAS & $\begin{array}{l}-0.21(-1.05, \\
0.62)\end{array}$ \\
\hline
\end{tabular}


Pain Physician: November/December 2016: 19:521-535

Table 1. Main characteristics of the studies (continued).

\begin{tabular}{|c|c|c|c|c|c|c|c|c|c|}
\hline \multicolumn{10}{|c|}{ Intervention Studies } \\
\hline Study (year) & Country & $\begin{array}{l}\text { Intervention } \\
\text { period }\end{array}$ & Disease & $\begin{array}{l}\text { Sample size } \\
\text { (Treatment/ } \\
\text { Control) }\end{array}$ & Intervention & Daily dose & Comparison & $\begin{array}{l}\text { Outcome } \\
\text { score }\end{array}$ & $\begin{array}{l}\text { Standard } \\
\text { Mean } \\
\text { Difference } \\
(95 \% \mathrm{CI})\end{array}$ \\
\hline \multirow[t]{2}{*}{$\begin{array}{l}\text { Geusens et al } \\
\text { (1994) }\end{array}$} & \multirow[t]{2}{*}{ Belgium } & \multirow[t]{2}{*}{12 months } & \multirow[t]{2}{*}{$\begin{array}{l}\text { Rheumatoid } \\
\text { arthritis }\end{array}$} & $90(21 / 20)$ & $\begin{array}{l}\text { Fish oil + } \\
\text { olive oil }\end{array}$ & $\begin{array}{l}840 \mathrm{mg} \\
\text { EPA, 180 } \\
\text { mg DHA }\end{array}$ & \multirow[t]{2}{*}{ Olive oil } & \multirow[t]{2}{*}{$\begin{array}{l}\text { 5-point } \\
\text { score }\end{array}$} & $\begin{array}{l}-1.10(-1.76, \\
-0.44)\end{array}$ \\
\hline & & & & $90(19 / 20)$ & Fish oil & $\begin{array}{l}1680 \mathrm{mg} \\
\mathrm{EPA}, 360 \\
\mathrm{mg} \text { DHA }\end{array}$ & & & $\begin{array}{l}-1.89(-2.65, \\
-1.14)\end{array}$ \\
\hline $\begin{array}{l}\text { Leventhal et al } \\
\text { (1994) }\end{array}$ & USA & 24 weeks & $\begin{array}{l}\text { Rheumatoid } \\
\text { arthritis }\end{array}$ & $34(7 / 7)$ & $\begin{array}{l}\text { Blackcurrant } \\
\text { seed oil }\end{array}$ & $\begin{array}{l}1995 \mathrm{mg} \\
\text { GLA }\end{array}$ & Soybean oil & VAS & $\begin{array}{l}-0.85(-1.94, \\
0.24)\end{array}$ \\
\hline $\begin{array}{l}\text { Leventhal et al } \\
\text { (1993) }\end{array}$ & USA & 24 weeks & $\begin{array}{l}\text { Rheumatoid } \\
\text { arthritis }\end{array}$ & $37(14 / 13)$ & $\begin{array}{l}\text { Borage seed } \\
\text { oil }\end{array}$ & $\begin{array}{l}1400 \mathrm{mg} \\
\text { GLA }\end{array}$ & $\begin{array}{l}\text { Cotton seed } \\
\text { oil }\end{array}$ & VAS & $\begin{array}{l}-1.11(-1.92, \\
-0.30)\end{array}$ \\
\hline $\begin{array}{l}\text { Magaró et al } \\
\text { (1992) }\end{array}$ & Italy & 45 days & $\begin{array}{l}\text { Rheumatoid } \\
\text { arthritis }\end{array}$ & $20(10 / 10)$ & $\begin{array}{l}\text { Diclofenac + } \\
\Omega-3\end{array}$ & $\begin{array}{l}1600 \mathrm{mg} \\
\text { EPA, 1100 } \\
\text { mg DHA }\end{array}$ & Diclofenac & VAS & $\begin{array}{l}0.42(-0.46 \\
1.31)\end{array}$ \\
\hline $\begin{array}{l}\text { Nielsen et al } \\
\text { (1992) }\end{array}$ & Denmark & 12 weeks & $\begin{array}{l}\text { Rheumatoid } \\
\text { arthritis - visual } \\
\text { pain }\end{array}$ & $57(27 / 24)$ & Fish oil & $\begin{array}{l}2000 \mathrm{mg} \\
\text { EPA, } 1200 \\
\text { mg DHA }\end{array}$ & $\begin{array}{l}\text { Fat } \\
\text { composition } \\
\text {; average } \\
\text { Danish diet }\end{array}$ & VAS & $\begin{array}{l}-0.58(-1.14, \\
-0.02)\end{array}$ \\
\hline $\begin{array}{l}\text { Sköldstam et al } \\
\text { (1992) }\end{array}$ & Sweden & 6 months & $\begin{array}{l}\text { Rheumatoid } \\
\text { arthritis }\end{array}$ & $46(22 / 21)$ & Fish oil & $\begin{array}{l}1800 \mathrm{mg} \\
\text { EPA, 1200 } \\
\text { mg DHA }\end{array}$ & $\begin{array}{l}\text { Inactive } \\
\text { oil (maize, } \\
\text { olive and } \\
\text { peppermint } \\
\text { oils) }\end{array}$ & $\begin{array}{l}\text { 4-point } \\
\text { score }\end{array}$ & $\begin{array}{l}0.04(-0.55 \\
0.64)\end{array}$ \\
\hline $\begin{array}{l}\text { Stammers et al } \\
\text { (1992) }\end{array}$ & $\begin{array}{l}\text { United } \\
\text { Kingdom }\end{array}$ & 6 months & Osteoarthritis & $86(29 / 29)$ & $\begin{array}{l}\text { Current } \\
\text { NSAIDs + } \\
\text { cod liver oil }\end{array}$ & $\begin{array}{l}786 \mathrm{mg} \\
\mathrm{EPA}\end{array}$ & $\begin{array}{l}\text { Current } \\
\text { NSAIDs + } \\
\text { olive oil }\end{array}$ & VAS & $\begin{array}{l}0.21(-0.31, \\
0.73)\end{array}$ \\
\hline $\begin{array}{l}\text { Brzeski et al } \\
\text { (1991) }\end{array}$ & $\begin{array}{l}\text { United } \\
\text { Kingdom }\end{array}$ & 6 months & $\begin{array}{l}\text { Rheumatoid } \\
\text { arthritis }\end{array}$ & $40(13 / 17)$ & EPO & $\begin{array}{l}540 \mathrm{mg} \\
\text { GLA }\end{array}$ & Olive oil & VAS & $\begin{array}{l}0.15(-0.57, \\
0.88)\end{array}$ \\
\hline \multirow[t]{2}{*}{$\begin{array}{l}\text { Kremer et al } \\
(1990)\end{array}$} & \multirow[t]{2}{*}{ USA } & \multirow[t]{2}{*}{24 weeks } & \multirow[t]{2}{*}{$\begin{array}{l}\text { Rheumatoid } \\
\text { arthritis }\end{array}$} & $64(20 / 12)$ & \multirow[t]{2}{*}{ Fish oil } & $\begin{array}{l}27 \text { and } 18 \\
\mathrm{mg} / \mathrm{kg} / \mathrm{day} \\
\text { of EPA and } \\
\text { DHA }\end{array}$ & \multirow[t]{2}{*}{ Olive oil } & \multirow[t]{2}{*}{$\begin{array}{l}5 \text {-point } \\
\text { score }\end{array}$} & \multirow[t]{2}{*}{$\begin{array}{l}0.23(-0.42, \\
0.88)\end{array}$} \\
\hline & & & & $64(17 / 12)$ & & $\begin{array}{l}54 \text { and } 36 \\
\mathrm{mg} / \mathrm{kg} / \text { day } \\
\text { of EPA and } \\
\text { DHA }\end{array}$ & & & \\
\hline $\begin{array}{l}\text { Van der } \\
\text { Tempel et al } \\
(1990)\end{array}$ & Netherlands & 3 months & $\begin{array}{l}\text { Rheumatoid } \\
\text { arthritis }\end{array}$ & $16(14 / 14)$ & $\begin{array}{l}\text { Fractionated } \\
\text { fish oil }\end{array}$ & $\begin{array}{l}2040 \mathrm{mg} \\
\mathrm{EPA}, 1320 \\
\mathrm{mg} \text { DHA }\end{array}$ & $\begin{array}{l}\text { Fractionated } \\
\text { coconut oil }\end{array}$ & VAS & $\begin{array}{l}-0.58(-1.33 \text {, } \\
0.18)\end{array}$ \\
\hline $\begin{array}{l}\text { Jäntti et al } \\
\text { (1989) }\end{array}$ & Finland & 12 weeks & $\begin{array}{l}\text { Rheumatoid } \\
\text { arthritis }\end{array}$ & $20(9 / 9)$ & $\begin{array}{l}\text { 20mL EPO } \\
\text { (9\% GLA) }\end{array}$ & $\begin{array}{l}1800 \mathrm{mg} \\
\text { GLA }\end{array}$ & Olive oil & VAS & $\begin{array}{l}0.25(-0.68 \\
1.17)\end{array}$ \\
\hline $\begin{array}{l}\text { Cleland et al } \\
\text { (1988) }\end{array}$ & Australia & 16 weeks & $\begin{array}{l}\text { Rheumatoid } \\
\text { arthritis }\end{array}$ & $60(23 / 23)$ & Fish oil & $\begin{array}{l}3200 \mathrm{mg} \\
\mathrm{EPA}, 2000 \\
\mathrm{mg} \text { DHA }\end{array}$ & Olive oil & VAS & $\begin{array}{l}-0.02(-0.60 \text {, } \\
0.56)\end{array}$ \\
\hline $\begin{array}{l}\text { Kremer et al } \\
\text { (1987) }\end{array}$ & USA & 14 weeks & $\begin{array}{l}\text { Rheumatoid } \\
\text { arthritis }\end{array}$ & $40(33 / 33)$ & Fish oil & $\begin{array}{l}2700 \mathrm{mg} \\
\mathrm{EPA}, 1800 \\
\mathrm{mg} \text { DHA }\end{array}$ & Placebo & $\begin{array}{l}\text { 5-point } \\
\text { score }\end{array}$ & $\begin{array}{l}-0.34(-0.82, \\
0.15)\end{array}$ \\
\hline
\end{tabular}

EPA: Eicosapentaenoic acid, DHA: Docosahexaenoic acid, GLA: Gamma-linolenic acid 
PUFA and Pain: A Meta-analysis

Table 2. Meta-analysis of intervention studies in chronic pain.

\begin{tabular}{|c|c|c|c|c|c|c|}
\hline & & $\begin{array}{l}\mathrm{N}^{\circ} \text { of } \\
\text { studies }\end{array}$ & $\begin{array}{l}\text { SMD Fixed effects [95\% } \\
\text { CI] }\end{array}$ & $\begin{array}{l}\text { SMD Random effects } \\
{[95 \% \mathrm{CI}]}\end{array}$ & Heterogeneity I 2 & $\begin{array}{c}Q \text {-test } \\
P \text {-value }\end{array}$ \\
\hline \multicolumn{2}{|l|}{ Main analysis } & 46 & $-0.37[-0.45,-0.30]$ & $-0.40[-0.58,-0.22]$ & 80.53 & 0.001 \\
\hline \multicolumn{7}{|l|}{ Subgroup } \\
\hline \multirow{4}{*}{ Disease } & Rheumatoid arthritis & 29 & $-0.34[-0.46,-0.23]$ & $-0,36[-0.62,-0.10]$ & 80.06 & 0.001 \\
\hline & Mastalgia & 5 & $0.03[-0.14,0.19]$ & $0.03[-0.14,0.19]$ & 0.00 & 0.94 \\
\hline & Dysmenorrhoea & 4 & $-0.85[-1.04,-0.66]$ & $-0.82[-1.21,-0.43]$ & 73.72 & 0.01 \\
\hline & Others diseases & 8 & $-0.51[-0.69,-0.32]$ & $-0.61[-1.03,-0.20]$ & 77.03 & 0.001 \\
\hline \multirow{4}{*}{ Fatty acid type } & $\Omega-3$ (from fish oil) & 27 & $-0.49[-0.59,-0.39]$ & $-0.47[-0.68,-0.26]$ & 76.50 & 0.001 \\
\hline & Gammalinolenic acid & 9 & $-0.08[-0.24,0.08]$ & $-0.16[-0.44,0.12]$ & 56.19 & 0.02 \\
\hline & Combined PUFA & 3 & $-0.39[-0.76,-0.02]$ & $-0.61[-1.83,0.60]$ & 90.32 & 0.001 \\
\hline & Dietary intervention & 5 & $-0.60[-0.84,-0.36]$ & $-0.63[-1.30,0.05]$ & 87.06 & 0.001 \\
\hline \multirow{2}{*}{$\begin{array}{l}\text { Daily dose } \\
\text { (overall) }\end{array}$} & $\leq 1.35 \mathrm{~g}$ & 22 & $-0.47[-0.56,-0.37]$ & $-0.55[-0.79,-0.30]$ & 83.40 & 0.001 \\
\hline & $>1.35 \mathrm{~g}$ & 20 & $-0.23[-0.37,-0.09]$ & $-0.29[-0.56,-0.03]$ & 69.90 & 0.001 \\
\hline \multirow{2}{*}{$\begin{array}{l}\text { Daily dose } \\
(\Omega-3)\end{array}$} & $\leq 1.35 \mathrm{~g}$ & 17 & $-0.70[-0.81,-0.58]$ & $-0.74[-1.01,-0.48]$ & 79.30 & 0.001 \\
\hline & $>1.35 \mathrm{~g}$ & 16 & $-0.16[-0.31,-0.01]$ & $-0.21[-0.49,0.07]$ & 70.40 & 0.001 \\
\hline \multirow{2}{*}{$\begin{array}{l}\text { Intervention } \\
\text { period (overall) }\end{array}$} & $\leq 3$ months & 21 & $-0.64[-0.75,-0.52]$ & $-0.56[-0.86,-0.25]$ & 84.02 & 0.001 \\
\hline & $>3$ months & 25 & $-0.18[-0.28,-0.07]$ & $-0.24[-0.43,-0.06]$ & 66.56 & 0.001 \\
\hline \multirow{2}{*}{$\begin{array}{l}\text { Intervention } \\
\text { period }(\Omega-3)\end{array}$} & $\leq 3$ months & 17 & $-0.83[-0.96,-0.70]$ & $-0.78[-1.04,-0.52]$ & 73.06 & 0.001 \\
\hline & $>3$ months & 18 & $-0.20[-0.32,-0.07]$ & $-0.24[-0.47,-0.01]$ & 69.15 & 0.001 \\
\hline \multirow{2}{*}{ Outcome score } & Composite score & 6 & $-0.41[-0.54,-0.27]$ & $-0.58[-1.07,-0.09]$ & 90.92 & 0.001 \\
\hline & VAS and Likert score & 40 & $-0.36[-0.45,-0.27]$ & $-0.37[-0.57,-0.17]$ & 77.81 & 0.001 \\
\hline \multirow{2}{*}{ Jadad score } & $<3$ & 10 & $-0.45[-0.64,-0.27]$ & $-0.38[-0.73,-0.03]$ & 68.32 & 0.001 \\
\hline & $\geq 3$ & 36 & $-0.36[-0.44,-0.27]$ & $-0.41[-0.62,-0.20]$ & 82.66 & 0.001 \\
\hline
\end{tabular}

toid arthritis, used $\Omega-3$ as an intervention, VAS as assessment score for pain, and had low risk of bias according to Jadad score. We retrieved 29 studies on rheumatoid arthritis, 5 studies on mastalgia, 4 studies on dysmenorrhea, and 8 studies on other diseases (osteoarthritis, migraines, asthenopia, chronic myalgia, myofascial pain syndrome). The data from one study were obtained from the authors (9). The main characteristics of the intervention studies are shown in Table 1.

PUFA supplementation was associated with a meaningful reduction in the risk of chronic pain (random effects pooled SMD $=-0.40,95 \% \mathrm{Cl}-0.58$ to -0.22 ).

Subgroup analyses (Table 2 and Fig. 2) show that supplementation of PUFA decreased the risk of all painful diseases except mastalgia. The strongest effect was observed for the 4 studies of dysmenorrhoea (SMD $-0.82,95 \% \mathrm{Cl}-1.21$ to -0.43$)$. The "other diseases" group showed moderate effect (SMD $-0.61,95 \% \mathrm{Cl}-1.03$ to -0.20), and the rheumatoid arthritis group small to moderate effect (SMD $-0.36,95 \% \mathrm{Cl}-0.62$ to, -0.10 ).

Heterogeneity was substantial overall and similarly high after stratification by design, quality features (including adjustment for confounders), and study population. Given the substantial heterogeneity, we focused on the random effects analysis. The fixed effects results are presented for comparison and discussed only where they differ.

When we stratified our analysis by type of fatty acid supplementation, the only subgroup that shows an association with pain is the $\Omega-3$ supplementation (SMD $-0.47,95 \% \mathrm{Cl}-0.68$ to -0.26$)$. The "combined PUFA" and "dietary intervention" groups show stronger effects, although not statistically significant using the random effects model but significant under the fixed effects model. The $\Omega-6$ group showed no effect.

For the analysis of the $\Omega-3$ group, we excluded one study which used conjugated linoleic acid (8), as it is a precursor and not strictly a $\Omega$-3PUFA. The results after including this study did not change meaningfully (SMD $-0.46,95 \% \mathrm{Cl}-0.67$ to -0.25$)$.

The stratification by daily dose of fatty acids and duration of the supplementation provided interesting 


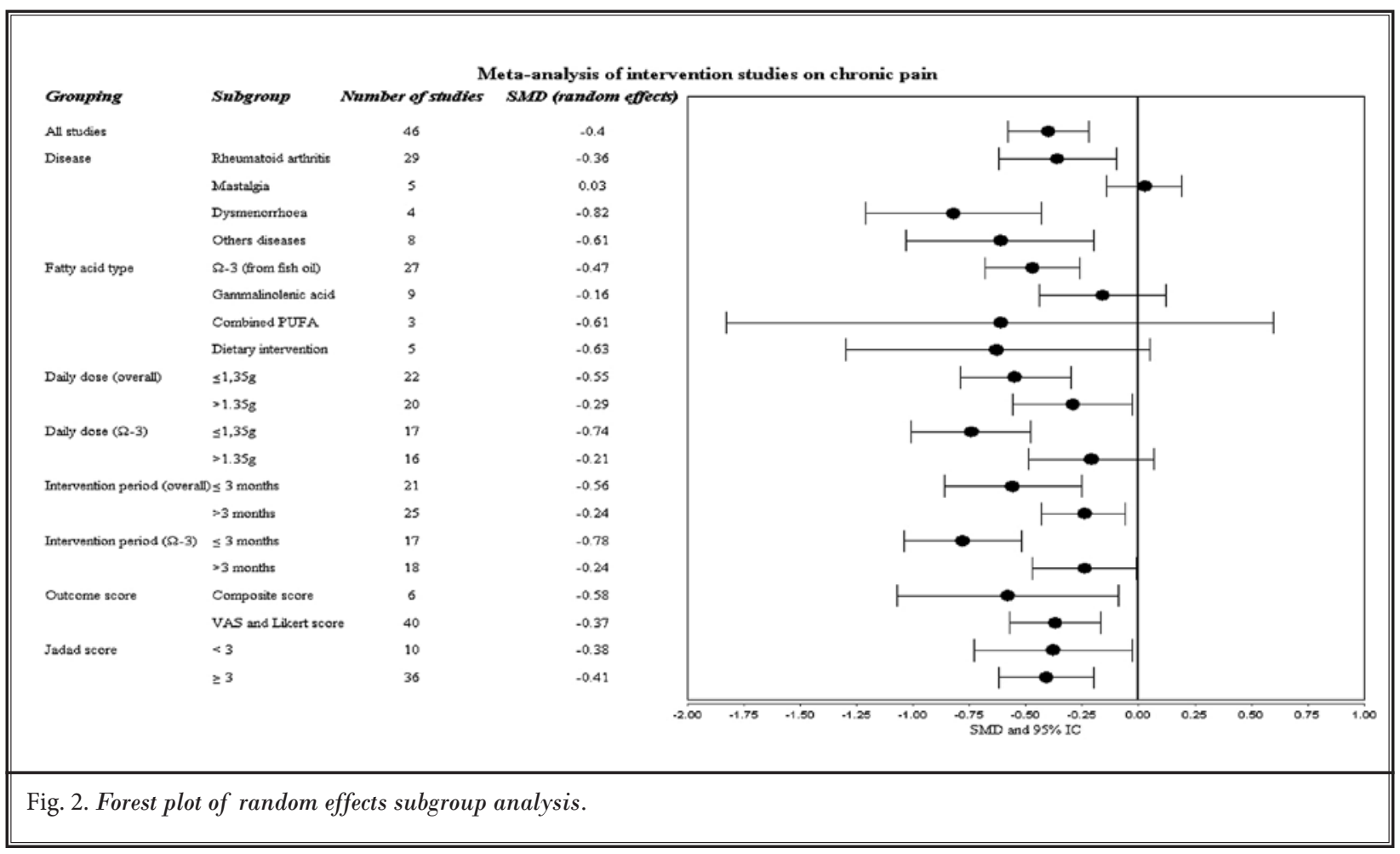

information. Compared to placebo, we observed a stronger effect for the low dose group, i.e., daily intake $\leq 1.35 \mathrm{~g}$ (SMD $-0.55,95 \% \mathrm{Cl}-0.79$ to -0.30 ) than for the high dose group i.e., daily intake > 1.35g (SMD -0.29, $95 \% \mathrm{Cl}-0.56$ to -0.03$)$. As for the intervention period, we found a larger effect for the $\leq 3$ months group (SMD $-0.56,95 \% \mathrm{Cl}-0.86$ to -0.25$)$ than for the $>3$ months group (SMD $-0.24,95 \% \mathrm{Cl}-0.43$ to -0.06 ).

Furthermore, when we restricted this doseresponse analysis to $\Omega-3$ intervention, again, the effect was larger for the low dose group (SMD $-0.74,95 \% \mathrm{Cl}$ -1.01 to -0.48 ) than for the high dose group, in which no effect was observed (SMD $-0.21,95 \% \mathrm{Cl}-0.49$ to 0.07 ). As in the general analysis, the effect was stronger for short interventions (SMD $-0.78,95 \% \mathrm{Cl}-1.04$ to -0.52 ) than for longer ones (> 3 months) (SMD $-0.24,95 \% \mathrm{Cl}$ -0.47 to -0.01 ).

Studies that used composite scores showed a stronger effect than studies that used VAS or Likert scores (SMD $-0.58,95 \% \mathrm{Cl}-1.07$ to -0.09 and $-0.37,95 \%$ $\mathrm{Cl}-0.57$ to -0.17 , respectively). No substantial difference was observed when we stratified the analysis by quality score (SMD $-0.38,95 \% \mathrm{Cl}-0.73$ to -0.03 for low quality studies and $-0.41,95 \% \mathrm{Cl}-0.62$ to -0.20 for high quality studies).

\section{Publication Bias}

The funnel plot of the effect sizes of the studies was slightly skewed to the left (Fig. 3). However, the more formal Egger's test showed no evidence for publication bias $(P=0.30)$. No studies were imputed in the Trim and Fill procedure when the random effects model was used, while 3 studies were imputed when the fixed effects model was used, resulting in a mean effect size of $-0.31(95 \% \mathrm{Cl}-0.50$ to -0.12$)$.

\section{Cumulative Meta-analysis}

We performed a cumulative meta-analysis of the 46 trials of pain and PUFA supplementation by year of publication (data not shown). A statistically significant effect was first observed after publication of the trial by Geusens et al in 1994 (53) (SMD -0.35, 95\% Cl -0.68 to -0.02 ) and at the same point when we considered pain from rheumatoid arthritis only. Subsequent trials have increased the precision of the effect but no meaningful changes were observed in the magnitude.

\section{Discussion}

The results of our meta-analysis of intervention studies support the hypothesis that PUFA supplementation improves chronic pain moderately. The effect is 


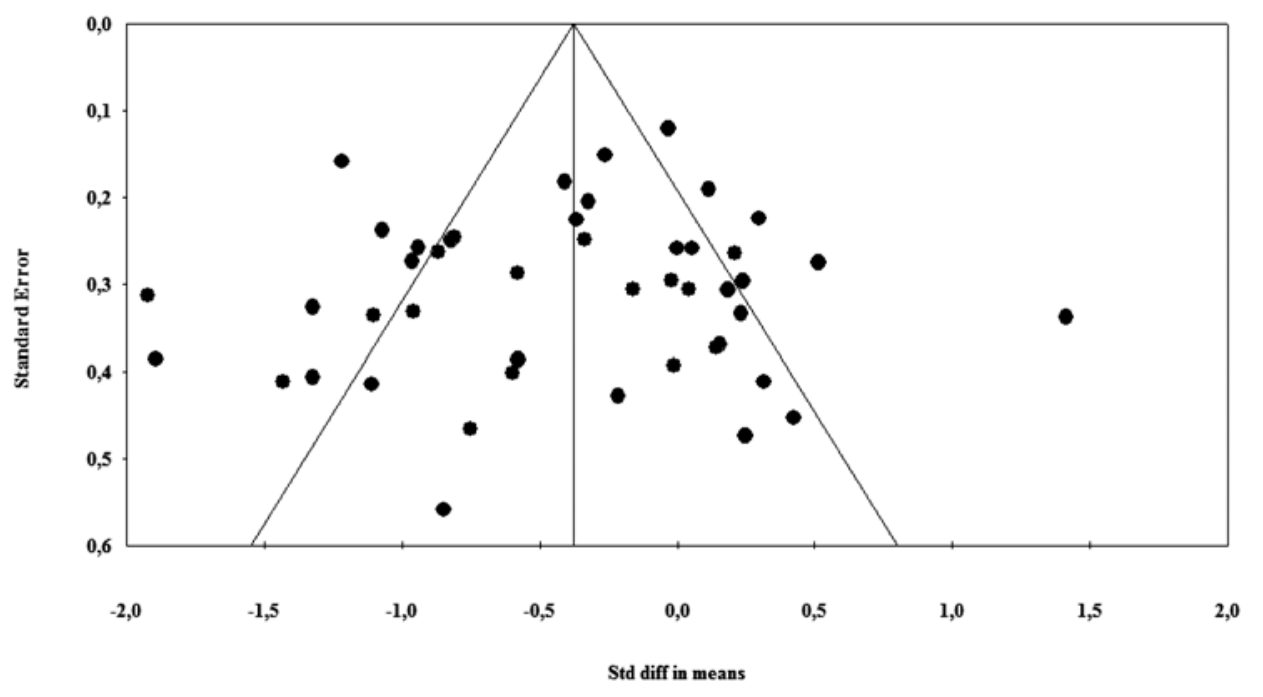

Fig. 3. Funnel plot of standard error by standard mean difference.

important for dysmenorrhoea and the group of "other chronic pain syndromes," less intense for rheumatoid arthritis, and absent for mastalgia. Apparently, this effect is mainly due to $\Omega-3$, as other types of supplementation failed to exert any effect. A larger effect was observed for low doses and short periods of treatment. The small number of observational studies available did not shed any light on a potential preventive effect of fatty acids on chronic pain and only one study (crosssectional) out of 5 was supportive of an association.

Publication bias could have affected the results of our study. This may have occurred if trials with nonsignificant or negative results would have remained unpublished. However, both funnel plot examination and Egger's test showed no evidence of publication bias, and the Trim-and-Fill method did not alter our results. We had to exclude 12 studies (Fig. 1) from those retrieved in our search as they were lacking essential data in order to be included in a meta-analysis, such as mean change in one or both groups $(66,67)$, standard deviation (68) or both values (69). Our efforts to obtain these data from the authors were in vain as the large majority could not be traced. The overall conclusions of those 12 studies swayed between beneficial effects of fatty acids to harmful ones. Theoretically, inclusion of these studies could then have modified our results. Nonetheless, this possibility is highly improbable in view of the results of the "Fail-safe N" procedure that we performed subsequently. The Fail-safe $\mathrm{N}$ procedure computes the number of studies with null results necessary to reverse the observed association in a metaanalysis and render it statistically non-significant (70). In our case this number was 982 . This number is too large for our results to be due to publication bias.

A set of meta-analyses with a limited number of studies, relating fatty acids supplementation to specific painful diseases, was published in the last decade. A meta-analysis of 9 studies, published in 2004, did not find any effect of $\Omega-3$ supplementation on patients assessed pain in rheumatoid arthritis (71). Another metaanalysis of 14 studies (12), published in 2007, focused on inflammatory pain and $\Omega-3$ supplementation and found a small to moderate improvement in pain-related outcomes. Finally, in 2012, Lee et al (72) published a meta-analysis of 10 studies restricted to high dose (> $2.7 \mathrm{~g} /$ day) and long period (> 3 months) $\Omega-3$ supplementation in rheumatoid arthritis. The authors did not find any effect of this supplementation scheme. The outcome used in these meta-analyses was generally a proxy of pain, such as inflammatory responses, physician evaluations, or non-steroidal anti-inflammatory drugs (NSAIDs) consumption. In our meta-analysis, we included only those studies that provided direct pain measurements, such as VAS or composite scores. The larger number of studies, the inclusion of $\Omega-6$ supplementation trials, and different dosages, intervention 
periods, and diseases can explain the differences and discrepancies between the results of those previous meta-analyses and our study.

The relation between PUFA and inflammation (and its concomitant pain) is well established. Eicosanoids such as prostaglandins (PG), thromboxanes, and leukotrienes (LTs) are important mediators and regulators of inflammation. They are synthesized through the cyclooxygenase (COX) and lypoxygenase (LOX) pathways from arachidonic acid (ARA), an $\Omega-6$ PUFA obtained from dietary sources and synthesis from linoleic and $\gamma$-linoleic acid (5). Inhibition of the COX pathway is the main action of NSAIDs. The $\Omega-3$ PUFAs EPA and DHA inhibit the ARA pathway, leading to decreased production of eicosanoids and other inflammatory cytokines such as TNF- $\alpha$, IL 1 , and IL 6 . Additionally, this inhibition gives rise to the synthesis of a series of leukotrienes with low inflammatory potential which may even act as antagonists of the ARA-derived mediators $(6,7)$. Furthermore, experts consider that DHA and EPA may give rise to powerful anti-inflammatory molecules, the resolvins $E 1$ and D1, which have analgesic effects (73).

S-6 PUFAs, in contrast, were generally considered to be involved in the molecular mechanism that induces pain, as prostaglandins, inflammatory eicosanoids, and cytokines derive from them. Nevertheless, some studies reported that $\Omega-6$ PUFAs are associated with the suppression of pain and may then both inhibit and induce pain transmission (7).

Our study has some limitations. We found high heterogeneity in almost all analyses. Some degree of heterogeneity was expected, as our meta-analysis includes trials of pain outcomes from different diseases, with different supplementation patterns, doses, duration, baseline characteristics, etc. The subgroup analyses did not succeed in eliminating this heterogeneity except for the case of mastalgia, which included a small group of trials with similar characteristics.

Another limitation is the fact that, in some studies, olive oil was used as placebo. Olive oil was shown to have anti-inflammatory properties, due to the fact that oleic acid competes with arachidonic acid (74). This could have reduced the effect observed in our meta-analysis. This potential dilution effect is confirmed when we stratified our analysis by placebo type. The 38 studies that did not use olive oil in the control group showed a more intense effect of PUFA (SMD $-0.43,95 \% \mathrm{Cl}-0.63$ to -0.23 ) than the 8 studies that used olive oil (SMD $-0.24,-0.68$ to 0.20 ). Our results would be biased towards the null value, which means that the effect of PUFA intake observed in our meta-analysis is underestimated.

The results of our meta-analysis show that high doses and long periods of supplementation are less effective in mitigating pain than low doses and short periods, especially in the $\Omega-3$ group. In their metaanalysis Goldberg and Katz (12) found that long treatment periods were effective only for physician-assessed pain, and not for other outcomes, but former reviews of clinical trials, ex vivo studies, and mice models about inflammatory diseases and $\Omega-3$ supplementation did show a dose and time-dependent relation, and ended up recommending high doses of fatty acids for long periods $(5,6)$. The explanation of our finding and thus, the reason for this apparent lack of consistency could be threefold: 1) previous meta-analyses measure outcomes that are different from ours. While our meta-analysis assessed pain as a symptom perceived by patients, those meta-analyses measured outcomes that could be considered as proxies of pain: morning stiffness, number of painful or tender joints, and NSAID consumption, among others. Recent research showed that $\Omega-3$ PUFA could relieve pain not only via immune regulation and inflammation suppression, but also via pain signalling inhibition and release of $\beta$-endorphins and resolvins (7). It is then plausible that the dose-response pattern varies, depending on whether subjective pain is used as an outcome, as in our meta-analysis, or another less subjective inflammation-related outcome. 2) In longer studies, patients referred decreasing their intake of NSAIDS (72). This could result in less pain relief. 3) Some placebo effect cannot be ruled out for shorter studies. A recent study suggested that the magnitude of the placebo effect could be larger in short studies than in long ones (75).

\section{Conclusion}

The large number of studies included, the magnitude of the associations found, the consistency of the results through different settings, and the existence of a mechanism that gives biologic plausibility to the relationship, provide evidence that PUFA supplementation may be moderately effective against pain, especially that associated with rheumatoid arthritis and dysmenorrhea. Existing studies on the potential preventive effect of PUFA intake are too scarce to lead to any conclusion. Future research should include careful evaluation of the dose-response relationship in clinical trials and implementation of cohort studies to assess preventive effects of PUFAs. 


\section{References}

1. International Association for the Study of Pain. Unrelieved pain is a major global healthcare problem. www.iasp-pain. org/files/Content/ContentFolders/Glob alYearAgainstPain2/20042005RighttoPa inRelief/factsheet.pdf. Accessed August 20, 2015 .

2. World Health Organization. The Global Burden of Disease: 2004 Update. Geneva, Switzerland: World Health Organization, 2008.

3. Tracey I, Bushnell MC. How neuroimaging studies have challenged us to rethink: Is chronic pain a disease? J Pain 2009; 10:1113-1120.

4. Gaskin DJ, Richard P. The economic costs of pain in the United States. J Pain 2012; 13:715-724.

5. Stamp LK, James MJ, Cleland LG. Diet and rheumatoid arthritis: A review of the literature. Semin Arthritis Rheum 2005; 35:77-94.

6. Miles EA, Calder PC. Influence of marine $n-3$ polyunsaturated fatty acids on immune function and a systematic review of their effects on clinical outcomes in rheumatoid arthritis. Br J Nutr 2012; 107:S171-S184.

7. Tokuyama S, Nakamoto K. Unsaturated fatty acids and pain. Biol Pharm Bull 2011; 34:1174-1178.

8. Aryaeian N, Shahram F, Djalali M, Eshragian MR, Djazayeri A, Sarrafnejad A, Salimzadeh A, Naderi N, Maryam C. Effect of conjugated linoleic acids, vitamin $E$ and their combination on the clinical outcome of Iranian adults with active rheumatoid arthritis. Int ] Rheum Dis 2009; 12:20-28.

9. Adam O, Beringer C, Kless T, Lemmen C, Adam A, Wiseman M, Adam P, Klimmek R, Forth W. Anti-inflammatory effects of a low arachidonic acid diet and fish oil in patients with rheumatoid arthritis. Rheumatol Int 2003; 23:27-36.

10. Ruano C, Henriquez P, Bes-Rastrollo M, Ruiz-Canela M, del Burgo CL, SánchezVillegas A. Dietary fat intake and quality of life: The SUN project. Nutr J 2011; 10:121.

11. Deutch B. Menstrual pain in Danish women correlated with low n-3 polyunsaturated fatty acid intake. Eur J Clin Nutr 1995; 49:508-516.

12. Goldberg RJ, Katz J. A meta-analysis of the analgesic effects of omega-3 polyunsaturated fatty acid supplementation for inflammatory joint pain. Pain 2007;
129:210-223.

13. Moher D, Liberati A, Tetzlaff J, Altman DG; PRISMA Group. Preferred reporting items for systematic reviews and metaanalyses: The PRISMA statement. PLoS Med 2009; 6:e1000097.

14. Booth A, Clarke M, Dooley G, Ghersi D, Moher D, Petticrew M, Stewart L. The nuts and bolts of PROSPERO: An international prospective register of systematic reviews. Syst Rev 2012; 1:2.

15. van der Windt D. The symptom of pain in populations. In: Croft P, Blyth FM, van der Windt D (eds). Chronic Pain Epidemiology. Oxford University Press, Oxford, 2010, pp 131-151.

16. Calder PC. n-3 polyunsaturated fatty acids, inflammation, and inflammatory diseases. Am J Clin Nutr 2006; 83:1505S-1519S.

17. International Association for the Study of Pain. Classification of Chronic Pain: Descriptions of Chronic Pain Syndromes and Definitions of Pain Terms. 2nd ed. Merskey H, Bogduk N (eds). IASP Press, Seattle, 1994.

18. Kawabata F, Tsuji T. Effects of dietary supplementation with a combination of fish oil, bilberry extract, and lutein on subjective symptoms of asthenopia in humans. Biomed Res 2011; 32:387-393.

19. Jadad AR, Moore RA, Carroll D, Jenkinson C, Reynolds DJ, Gavaghan DJ, McQuay HJ. Assessing the quality of reports of randomized clinical trials: Is blinding necessary? Control Clin Trials 1996; 17:1-12.

20. Faraone SV. Interpreting estimates of treatment effects: Implications for managed care. PT 2008; 33:700-711.

21. Rees D, Miles EA, Banerjee T, Wells SJ, Roynette CE, Wahle KW, Calder PC. Dose-related effects of eicosapentaenoic acid on innate immune function in healthy humans: A comparison of young and older men. Am J Clin Nutr 2006; 83:331-342.

22. Egger M, Davey Smith G, Schneider M, Minder C. Bias in meta-analysis detected by a simple, graphical test. BMJ 1997; 315:629-634.

23. Duval S, Tweedie R. Trim and fill: A simple funnel-plot-based method of testing and adjusting for publication bias in meta-analysis. Biometrics 2000; 56:455-463.

24. Nagata C, Hirokawa K, Shimizu N, Shimizu $\mathrm{H}$. Soy, fat and other dietary fac- tors in relation to premenstrual symptoms in Japanese women. BJOG 2004; 111:594-599.

25. Nagata C, Hirokawa K, Shimizu N, Shimizu $\mathrm{H}$. Associations of menstrual pain with intakes of soy, fat and dietary fiber in Japanese women. Eur J Clin Nutr 2005; 59:88-92.

26. Di Cintio E, Parazzini F, Tozzi L, Luchini L, Mezzopane R, Marchini M, Fedele L. Dietary habits, reproductive and menstrual factors and risk of dysmenorrhoea. Eur J Epidemiol 1997; 13:925-930.

27. Cortes ML. Aspectos clínico-nutricionais da suplementação de ácidos graxos ômega 3 em portadores de Síndrome Dolorosa Miofascial. July 2014. [Master's Dissertation] http://repositorio.ufba.br/ ri/handle/ri/15201. Accessed August 20, 2015.

28. Park Y, Lee A, Shim S-C, Lee JH, Choe JY, Ahn H, Choi CB, Sung YK, Bae SC. Effect of $n-3$ polyunsaturated fatty acid supplementation in patients with rheumatoid arthritis: A 16-week randomized, double-blind, placebo-controlled, parallel-design multicenter study in Korea. J Nutr Biochem 2013; 24:1367-1372.

29. Sohrabi N, Kashanian M, Ghafoori SS, Malakouti SK. Evaluation of the effect of omega- 3 fatty acids in the treatment of premenstrual syndrome: "A pilot trial." Complement Ther Med 2013; 21:141-146.

30. Ramsden CE, Faurot KR, Zamora D, Suchindran CM, Macintosh BA, Gaylord S, Ringel A, Hibbeln JR, Feldstein AE, Mori TA, Barden A, Lynch C, Coble R, Mas E, Palsson O, Barrow DA, Mann JD. Targeted alteration of dietary n-3 and $n-6$ fatty acids for the treatment of chronic headaches: A randomized trial. Pain 2013; 154:2441-2451.

31. Caturla N, Funes L, Pérez-Fons L, Micol V. A randomized, double-blinded, placebo-controlled study of the effect of a combination of lemon verbena extract and fish oil omega-3 fatty acid on joint management. J Altern Complement Med 2011; 17:1051-1063.

32. Guimarães KAG, Guimarães JP, Raposo NRB. Influência da suplementação alimentar com ômega 3 na remissão da mialgiacrônica centralmente mediada. Rev Gaucha Odontol 2011; 59:555-563.

33. Rahbar N, Asgharzadeh N, Ghorbani R. Effect of omega-3 fatty acids on intensity of primary dysmenorrhea. Int ] Gynaecol Obstet 2012; 117:45-47. 
34. Moghadamnia AA, Mirhosseini N, Abadi $\mathrm{MH}$, Omranirad A, Omidvar S. Effect of Clupeonella grimmi (anchovy/kilka) fish oil on dysmenorrhoea. East Mediterr Health J 2010; 16:408-413.

35. Pruthi S, Wahner-Roedler DL, Torkelson C), Cha SS, Thicke LS, Hazelton JH, Bauer BA. Vitamin $E$ and evening primrose oil for management of cyclical mastalgia: A randomized pilot study. Altern Med Rev 2010; 15:59-67.

36. Gupta $A B$ Das, Hossain AKMM, Islam $\mathrm{MH}$, Dey SR, Khan AL. Role of omega-3 fatty acid supplementation with indomethacin in suppression of disease activity in rheumatoid arthritis. Bangladesh Med Res Counc Bull 2009; 35:63-68.

37. Gruenwald J, Petzold E, Busch R, Petzold H-P, Graubaum H-J. Effect of glucosamine sulfate with or without omega-3 fatty acids in patients with osteoarthritis. Adv Ther 2009; 26:858-871.

38. Galarraga B, Ho M, Youssef HM, Hill A, McMahon H, Hall C, Ogston S, Nuki G, Belch JJ. Cod liver oil (n-3 fatty acids) as a non-steroidal anti-inflammatory drug sparing agent in rheumatoid arthritis. Rheumatology (Oxford) 2008; 47:665-669.

39. Goyal A, Mansel RE;Efamast Study Group. A randomized multicenter study of gamolenic acid (Efamast) with and without antioxidant vitamins and minerals in the management of mastalgia. Breast J 2005; 11:41-47.

40. Berbert AA, Kondo CRM, Almendra CL, Matsuo T, Dichi I. Supplementation of fish oil and olive oil in patients with rheumatoid arthritis. Nutrition 2005; 21:131-136.

41. Sundrarjun T, Komindr S, Archararit N, Dahlan W, Puchaiwatananon O, Angthararak S, Udomsuppayakul U, Chuncharunee $S$. Effects of $n-3$ fatty acids on serum interleukin-6, tumour necrosis factor-alpha and soluble tumour necrosis factor receptor $\mathrm{p} 55$ in active rheumatoid arthritis. J Int Med Res 2004; 32:443-454.

42. Remans PHJ, Sont JK, Wagenaar LW, Wouters-Wesseling W, Zuijderduin WM, Jongma A, Breedveld FC, Van Laar JM. Nutrient supplementation with polyunsaturated fatty acids and micronutrients in rheumatoid arthritis: Clinical and biochemical effects. Eur J Clin Nutr 2004; 58:839-845.

43. Sampalis F, Bunea R, Pelland MF, Kowalski O, Duguet N, Dupuis S. Evaluation of the effects of Neptune Krill Oil on the management of premenstrual syndrome and dysmenorrhea. Altern Med
Rev 2003; 8:171-179.

44. Santos ALG dos, Filho F, S AS, da Costa AM, Araújo RRF. Comparação entre óleo de borragem e óleo de girassol quanto à eficácia no tratamento de mulheres com mastalgia cíclica. Rev Bras Mastol 2005; 15:119-129.

45. Blommers J, de Lange-De Klerk ES m, Kuik DJ, Bezemer PD, Meijer S. Evening primrose oil and fish oil for severe chronic mastalgia: A randomized, double-blind, controlled trial. Am J Obstet Gynecol 2002; 187:1389-1394.

46. Harel Z, Gascon G, Riggs S, Vaz R, Brown W, Exil G. Supplementation with omega-3 polyunsaturated fatty acids in the management of recurrent migraines in adolescents. J Adolesc Health 2002; 31:154-161.

47. Volker D, Fitzgerald P, Major G, Garg M. Efficacy of fish oil concentrate in the treatment of rheumatoid arthritis. J Rheumatol 2000; 27:2343-2346.

48. Sarzi-Puttini P, Comi D, Boccassini L, Muzzupappa S, Turiel M, Panni B, Salvaggio $A$. Diet therapy for rheumatoid arthritis. A controlled double-blind study of two different dietary regimens. Scand J Rheumatol 2000; 29:302-307.

49. Hansen GV, Nielsen L, Kluger E, Thysen $M$, Emmertsen $H$, Stengaard-Pedersen K, Hansen EL, Unger B, Andersen PW. Nutritional status of Danish rheumatoid arthritis patients and effects of a diet adjusted in energy intake, fish-meal, and antioxidants. Scand J Rheumatol 1996; 25:325-330.

50. Zurier RB, Rossetti RG, Jacobson EW, DeMarco DM, Liu NY, Temming JE, White BM, Laposata M. gamma-Linolenic acid treatment of rheumatoid arthritis. A randomized, placebo-controlled trial. Arthritis Rheumatol 1996; 39:1808-1817.

51. Kremer JM, Lawrence DA, Petrillo GF, Litts LL, Mullaly PM, Rynes RI, Stocker RP, Parhami N, Greenstein NS, Fuchs $B R$. Effects of high-dose fish oil on rheumatoid arthritis after stopping nonsteroidal anti-inflammatory drugs. Clinical and immune correlates. Arthritis Rheumatol 1995; 38:1107-1114.

52. Nordström DC, Honkanen VE, Nasu Y, Antila E, Friman C, Konttinen YT. Alphalinolenic acid in the treatment of rheumatoid arthritis. A double-blind, placebo-controlled and randomized study: Flaxseed vs. safflower seed. Rheumatol Int 1995; 14:231-234.

53. Geusens P, Wouters C, Nijs J, Jiang Y,
Dequeker J. Long-term effect of omega-3 fatty acid supplementation in active rheumatoid arthritis. A 12-month, double-blind, controlled study. Arthritis Rheumatol 1994; 37:824-829.

54. Leventhal LJ, Boyce EG, Zurier RB. Treatment of rheumatoid arthritis with blackcurrant seed oil. $\mathrm{Br} J$ Rheumatol 1994; 33:847-852.

55. Leventhal LJ, Boyce EG, Zurier RB. Treatment of rheumatoid arthritis with gammalinolenic acid. Ann Intern Med 1993; 119:867-873.

56. Magarò $\mathrm{M}$, Zoli A, Altomonte L, Mirone L, De Sole P, Di Mario G, De Leo E. Effect of fish oil on neutrophil chemiluminescence induced by different stimuli in patients with rheumatoid arthritis. Ann Rheum Dis 1992; 51:877-880.

57. Nielsen GL, Faarvang KL, Thomsen BS, Teglbjaerg KL, Jensen LT, Hansen TM, Lervang HH, Schmidt EB, Dyerberg J, Ernst $E$. The effects of dietary supplementation with $n-3$ polyunsaturated fatty acids in patients with rheumatoid arthritis: A randomized, double blind trial. Eur J Clin Invest 1992; 22:687-691.

58. Sköldstam L, Börjesson $O$, Kjällman A, Seiving B, Akesson B. Effect of six months of fish oil supplementation in stable rheumatoid arthritis. A doubleblind, controlled study. Scand J Rheumatol 1992; 21:178-185.

59. Stammers T, Sibbald B, Freeling P. Efficacy of cod liver oil as an adjunct to non-steroidal anti-inflammatory drug treatment in the management of osteoarthritis in general practice. Ann Rheum Dis 1992; 51:128-129.

6o. Brzeski M, Madhok R, Capell HA. Evening primrose oil in patients with rheumatoid arthritis and side-effects of nonsteroidal anti-inflammatory drugs. $\mathrm{Br}$ J Rheumatol 1991; 30:370-372.

61. Kremer JM, Lawrence DA, Jubiz W, DiGiacomo R, Rynes R, Bartholomew LE, Sherman M. Dietary fish oil and olive oil supplementation in patients with rheumatoid arthritis. Clinical and immunologic effects. Arthritis Rheum 1990; 33:810-820.

62. van der Tempel H, Tulleken JE, Limburg PC, Muskiet FA, van Rijswijk MH. Effects of fish oil supplementation in rheumatoid arthritis. Ann Rheum Dis 1990; 49:76-80.

63. Jäntti J, Seppälä E, Vapaatalo $H$, Isomäki $\mathrm{H}$. Evening primrose oil and olive oil in treatment of rheumatoid arthritis. Clin Rheumatol 1989; 8:238-244. 
64. Cleland LG, French JK, Betts WH, Murphy GA, Elliott MJ. Clinical and biochemical effects of dietary fish oil supplements in rheumatoid arthritis. J Rheumatol 1988; 15:1471-1475.

65. Kremer JM, Jubiz W, Michalek A, Rynes RI, Bartholomew LE, Bigaouette J, Timchalk M, Beeler D, Lininger L. Fish-oil fatty acid supplementation in active rheumatoid arthritis. A double-blinded, controlled, crossover study. Ann Intern Med 1987; 106:497-503.

66. Harel Z, Biro FM, Kottenhahn RK, Rosenthal SL. Supplementation with omega-3 polyunsaturated fatty acids in the management of dysmenorrhea in adolescents. Am J Obstet Gynecol 1996; 174:1335-1338.

67. Kremer JM, Bigauoette J, Michalek AV, Timchalk MA, Lininger L, Rynes RI, Huyck C, Zieminski J, Bartholomew LE. Effects of manipulation of dietary fatty acids on clinical manifestations of rheumatoid arthritis Lancet 1985; 1:184-187.
68. Lau CS, Morley KD, Belch JJ. Effects of fish oil supplementation on non-steroidal anti-inflammatory drug requirement in patients with mild rheumatoid arthritis: A double-blind placebo controlled study. Br J Rheumatol 1993; 32:982-989.

69. Kjeldsen-Kragh J, Lund JA, Riise T, Finnanger B, Haaland K, Finstad R, Mikkelsen K, Førre O. Dietary omega-3 fatty acid supplementation and naproxen treatment in patients with rheumatoid arthritis. J Rheumatol 1992; 19:1531-1536.

70. Rosenthal R. The file drawer problem and tolerance for null results. Psychological Bulletin 1979; 86:638-641.

71. MacLean CH, Mojica WA, Morton SC Pencharz J, Hasenfeld Garland R, Tu W, Newberry SJ, Jungvig LK, Grossman J, Khanna P, Rhodes S, Shekelle P. Effects of omega-3 fatty acids on lipids and glycemic control in type II diabetes and the metabolic syndrome and on inflammatory bowel disease, rheumatoid arthritis, renal disease, systemic lupus erythema- tosus, and osteoporosis. Evid Rep Technol Assess (Summ) 2004; 89:1-4.

72. Lee YH, Bae SC, Song GG. Omega-3 polyunsaturated fatty acids and the treatment of rheumatoid arthritis: A meta-analysis. Arch Med Res 2012; 43:356-362.

73. Ji RR, Xu ZZ, Strichartz G, Serhan CN. Emerging roles of resolvins in the resolution of inflammation and pain. Trends Neurosci 2011; 34:599-609.

74. Moreno JJ, Carbonell T, Sánchez T, Miret S, Mitjavila MT. Olive oil decreases both oxidative stress and the production of arachidonic acid metabolites by the prostaglandin $\mathrm{G} / \mathrm{H}$ synthase pathway in rat macrophages. J Nutr 2001; 131(8):2145-2149.

75. Koog YH, Jung WY. Time course of placebo effect of acupuncture on pain: A systematic review. Int Sch Res Notices 2012; 2013:e204108. 
